\title{
Optical Multispectral Camera Communications Using LED Spectral Emission Variations
}

\author{
Daniel Moreno ${ }^{\circledR}$, Julio Rufo ${ }^{\circledR}$, Victor Guerra ${ }^{\circledR}$, Jose Rabadan, and Rafael Perez-Jimenez
}

\begin{abstract}
This work proposes using the changes in the LEDs' spectral emission induced by temperature variations to implement emitters for new communication channels in Optical Camera Communication (OCC) systems. If a camera can discriminate the shift in LEDs' spectral response, these modifications can be contemplated as a new data channel. The main advantage is that new channels can be obtained from the same physical optical emitter device, only temperature changes are needed. Thus, in this work, the use of multispectral or hyperspectral cameras as OCC receivers is proposed to increase the number of available communication channels, taking advantage of the high spectral resolutions of the camera devices.
\end{abstract}

Index Terms-OCC, multispectral, hyperspectral, LED, temperature dependence, peak wavelength.

\section{INTRODUCTION}

$\mathbf{O}$ PTICAL Camera Communication (OCC) has gained momentum in the last few years. The ubiquity of embedded optical cameras into consumer electronic devices such as Smart Phones, Tablets or laptops is expected to facilitate the mass adoption of this technology in the near future. In this sense, it is worth highlighting that this technology has been included in the revision of the IEEE 802.15.7 standard on Visible Light Communications [1], occupying a significant part of the document. Most recent OCC CMOS-based sensors allow high-speed communication due to the rolling shutter scanning mode; however, since OCC is based on image-forming optics, the maximum achievable speed depends on the light source's projected size [2].

On the other hand, multispectral cameras (and hyperspectral cameras) have been intensively used in several sectors such as agriculture [3], failure inspection in industry [4], and biotechnology [5]. Multispectral and hyperspectral cameras provide a higher number of communication bands, much more numerous than the three RGB channels available in conventional cameras. In this way, Optical Multispectral Camera

Manuscript received March 29, 2021; revised May 3, 2021; accepted May 6, 2021. Date of publication May 10, 2021; date of current version May 14, 2021. This work was supported in part by the Trainee Predoctoral Research Staff Program (2019) of the Universidad de Las Palmas de Gran Canaria, in part by the Canary Islands Regional Government under Project ATICCuA ProID2017010053, and in part by the Spanish Research Administration through the Ministerio de Ciencia e Innovación (MICINN) Project: Arquitecturas Ópticas Híbridas Para Ciudades Inteligentes (OSCAR) under Grant TEC 2017-84065-C3-1-R. (Corresponding author: Daniel Moreno.)

The authors are with the Institute for Technological Development and Innovation in Communications, Universidad de las Palmas de Gran Canaria 35017 Las Palmas de Gran Canaria, Spain (e-mail: dmoreno@idetic.eu).

Color versions of one or more figures in this letter are available at https://doi.org/10.1109/LPT.2021.3078842.

Digital Object Identifier 10.1109/LPT.2021.3078842
Communication (OMCC) opens up a range of possibilities in this area. Nevertheless, in order to carry out Wavelength Division Multiplexing (WDM), several devices presenting different peak wavelengths are usually needed. This leads to a channel-crosstalk compensation problem similar to the channel matrix estimation and inversion problem of Multiple Input Multiple Output (MIMO) systems.

Moreover, LED devices are affected by temperature. The most important effects are a variation on the emitted spectrum and a reduction of the luminous efficiency. The LED spectral changes induced by temperature variations are analyzed as a potentially positive phenomenon in this work. Taking advantage of the spectral richness of LED emissions and the significant number of bands that a multispectral (or hyperspectral) camera may present, several separable communication channels can be established using the same kind of device, reducing the system's complexity and cost.

In this letter, Section II presents the theoretical framework that supports the work. Sections III and IV present both the characterization methodology and results regarding the effects of temperature on the used LED devices. Furthermore, the application of this characterization is presented in Section V using a CMOS-based multispectral camera. Finally, several conclusions are extracted in Section VI.

\section{EFFECT OF TEMPERATURE ON LED}

The photons emitted by an LED present a wavelength that is related to the energy gap of the semiconductor substrate. In addition, the energy gap is affected by the p-n junction temperature, which is usually modeled using Equation 1. According to [6], in most semiconductor materials the energy gap diminishes as temperature increases. Therefore, as wavelength is inversely proportional to this gap, the peak wavelength of the LED increases with temperature.

$$
E_{g}=E_{0}-\frac{\alpha T^{2}}{T+\beta}
$$

where $T$ is temperature, $E_{0}$ is energy gap at $0 \mathrm{~K}$ temperature condition, and $\alpha$ and $\beta$ are semiconductor-dependent constants, which are empirically determined.

Nonetheless, the metallic content in the LED material must be taken into consideration. In [7], the authors demonstrated that depending on the Indium (In) content in InGaN alloys (green and blue LEDs), the band gap may increase with temperature. 
Furthermore, in general, the conversion efficiency decreases and the spectral width grows as temperature increases [8]. In this work, these generally undesired changes on the LED emission are grasped to improve communications by increasing the number of communication channels. Although these spectral variations may be subtle, the use of high spectral resolution cameras (such as multispectral cameras) is proposed as suitable and commercially-available receivers.

\section{Methodology}

The main objective of this letter is to take advantage of the spectral changes of the LED caused when its temperature increases, in the sense that the wavelength variation could be captured by a multispectral camera and then find an OCC application. This goal is achieved by carrying out the characterization of some LEDs to determine how the temperature affects their spectrum. In addition, those LEDs have been used in combination with a multispectral camera to form a communication link. Finally, some performance metrics regarding channel separability have been obtained to assess the feasibility of Optical Multispectral Camera Communications (OMCC), taking advantage of temperature-induced variations on the LEDs.

In order to induce different junction temperatures, the LEDs (common cathode RGB LED) were driven using currents ranging from $10 \mathrm{~mA}$ to $130 \mathrm{~mA}$. For each current, the junction temperature, the emitted spectrum, and the multispectral signature were obtained. Due to temperature diffusion effects, the system was stabilized for 5 minutes before acquiring the measurements mentioned above, in spite of the fact that the LEDs under test reached their thermally stable state after about 3 minutes. The devices were under ambient conditions, no external sources were employed to increase the LED's temperature and they were not externally thermally stabilized. Just their driving current was used to induce thermal variations owing to the Joule effect.

The experiments were carried out using the setups depicted in Figs. 1a and 1b. The devices under test were driven using an Ethernet-controlled current source (Yokogawa GS820). The LED junction temperature was measured using a thermographic camera (FLIR A645), while the emission spectra were obtained using a spectrometer (Spectral Products SM442). In order to complement this spectral measurement, the radiant power of each LED at the different tested currents was measured using an integrating sphere and an LED tester (Gigahertz BTS256).

Once the LEDs were characterized, a multispectral camera (SILIOS Technologies CMS-C1-C-EVR1M-GigE) was used to capture the wavelength variation. This 9 -bands camera ( 8 color narrow bands and 1 panchromatic band) covers the wavelength range from 400 to $1000 \mathrm{~nm}$. Those bands are the result of eight band-pass filters (narrow bands) and a filter sensitive to the entire wavelength range (panchromatic band). It has a CMOS sensor and a resolution of $1280(\mathrm{H}) \times 1024(\mathrm{~V})$ in the raw picture and $426(\mathrm{H}) \times 339(\mathrm{~V})$ for multispectral images. Furthermore, it was ensured that the images were not saturated, and the spectral signatures at several temperatures were extracted from them.

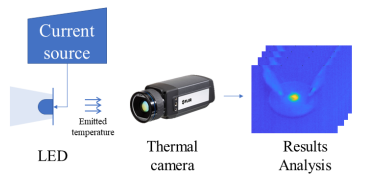

(a) Scheme used to get the temperature data.

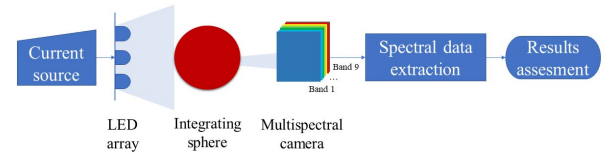

(b) Scheme used to get the spectral data

Fig. 1. System setup schemes.

Since the main objective of this work is to demonstrate the feasibility of grasping the spectral variation of LEDs with temperature, the channel separability of a combined emission using an arbitrary number of devices was evaluated using the condition number (Equation 2). This number is an algebraic metric that shows how small variations on a matrix may produce great variations on its inverse. In other words, this metric provides an indication as to whether several emissions can be separated as different channels. Well-conditioned matrices present condition numbers close to one, while ill-conditioned ones have high-value metrics.

$$
\operatorname{cond}(S)=\|S\| \cdot\left\|S^{-1}\right\|
$$

$S$ is the matrix comprising the spectral signatures. The spectral responses are added to the matrix $S$ so that it has one signature per column. Thus, the matrix dimension is $s b \times s r$, where $s b$ is the number of spectral bands (in this case, 9) and $s r$ is the number of signatures appended. This strategy has been proposed in VLC MIMO systems [9], and also in traditional RF MIMO works as a suitable mathematical tool. Additionally, condition number penalizes the signal-to-noise ratio (SNR) depending on the required spectral efficiency [10].

After obtaining the spectral signatures and the corresponding $S$ matrix, the OCC system performance was simulated assuming an On-Off Keying (OOK) transmission. This simulation involved a transmission signal composed of a bit stream $b$ (generated as a uniformly distributed pseudo-random sequence) and a normally distributed noise signal $n$, whose level depends on the selected SNR and the reference power (estimated from the relative maximum power inside $S$ ). Then, a reception process based on a zero-forcing (ZF) equalization (Equation 3) is applied to the transmitted signal for obtaining the received bit stream $\hat{b}$. Finally, the system's BER performance is evaluated for the considered SNR values (from $10 \mathrm{~dB}$ to $40 \mathrm{~dB}$ ). Sequences of $10^{6}$ bits per simulation were used, limiting the maximum accuracy to $10^{-6}$, and matrices $S$ with all the 2-channel combinations were assessed in order to analyze channel separability.

No different channel models have been considered in the simulation because the targeted-effect of temperature variations in a Tx-Rx link would not be affected by them.

$$
\hat{b}=(b \cdot S+n) \cdot S^{+}
$$

where $S^{+}$is the Moore-Penrose pseudoinverse of channel matrix $S$. 
TABLE I

SPECTRAL INFORMATION AT DIFFERENT TEMPERATURES

\begin{tabular}{|c|c|c|c|c|}
\hline LED & \multicolumn{4}{|c|}{ Red } \\
\hline Current $(\mathrm{mA})$ & $10\left(27^{\circ} \mathrm{C}\right)$ & $50\left(38^{\circ}{ }^{\circ}\right)$ & $100\left(56^{\circ} \mathrm{C}\right)$ & $130\left(65^{\circ} \mathrm{C}\right)$ \\
\hline Peak wavelength $(\mathrm{nm})$ & 659.6 & 663.6 & 672.5 & 680.7 \\
\hline FWHM $(\mathrm{nm})$ & 8.73 & 14.97 & 22.33 & 24.15 \\
\hline Peak spectral radiant power $(\mu \mathrm{W} / \mathrm{nm})$ & 2.75 & 10.53 & 12.33 & 8.29 \\
\hline
\end{tabular}

\begin{tabular}{|c|c|c|c|c|}
\hline LED & \multicolumn{4}{|c|}{ Green } \\
\hline Current $(\mathrm{mA})$ & $10\left(27^{\circ} \mathrm{C}\right)$ & $50\left(42^{\circ} \mathrm{C}\right)$ & $100\left(65^{\circ} \mathrm{C}\right)$ & $130\left(76^{\circ} \mathrm{C}\right)$ \\
\hline Peak wavelength $(\mathrm{nm})$ & 547.2 & 544.9 & 545.1 & 546.8 \\
\hline FWHM $(\mathrm{nm})$ & 23.73 & 31.80 & 40.92 & 43.18 \\
\hline Peak spectral radiant power $(\mu \mathrm{W} / \mathrm{nm})$ & 6.76 & 16.57 & 18.75 & 15.94 \\
\hline
\end{tabular}

\begin{tabular}{|c|c|c|c|c|}
\hline LED & \multicolumn{4}{|c|}{ Blue } \\
\hline Current $(\mathrm{mA})$ & $10\left(28^{\circ} \mathrm{C}\right)$ & $50\left(43^{\circ} \mathrm{C}\right)$ & $100\left(66^{\circ} \mathrm{C}\right)$ & $130\left(78^{\circ} \mathrm{C}\right)$ \\
\hline Peak wavelength $(\mathrm{nm})$ & 486.1 & 486.1 & 490.5 & 499.6 \\
\hline FWHM $(\mathrm{nm})$ & 16.68 & 29.62 & 45.85 & 32.10 \\
\hline Peak spectral radiant power $(\mu \mathrm{W} / \mathrm{nm})$ & 11.32 & 24.65 & 26.23 & 25.91 \\
\hline
\end{tabular}

\section{Characterization RESUlts}

Table I summarizes the temperature-induced effects on the LED emission. The peak wavelength, the Full Width at Half Maximum (FWHM) and the peak spectral radiant power values of each spectrum are shown. It can be observed that, in most cases, the emitted peak wavelength grew (the energy band gap decreases) as the junction temperature increased. The largest shift was obtained for the red LED with a difference of $21.1 \mathrm{~nm}$ for a temperature span of $38^{\circ} \mathrm{C}$. The blue LED presented a $13.5 \mathrm{~nm}$ shift for a temperature variation of $50{ }^{\circ} \mathrm{C}$. Finally, the measurements on the green LED showed that the peak wavelength slightly decreased with temperature. In this case, the highest peak wavelength was found at the minimum temperature. This behavior differs from the other colors' because of the metallic content in the green LED, as discussed in Section II.

Furthermore, it must be noted that the spectral width changed with temperature. The most remarkable variation was obtained in blue, in which the dissimilarity between the width at $28{ }^{\circ} \mathrm{C}$ and $66^{\circ} \mathrm{C}$ was approximately $30 \mathrm{~nm}$ (Table I).

Finally, concerning the spectral radiant power, the highest levels were obtained for the blue LED, followed by green and red. Note that this measurement did not take into account the electrical power, which would have shown that the red LED presents the highest quantum efficiency. Moreover, it can be noted that at the maximum temperature (driving current $130 \mathrm{~mA}$ ), the power decreases with respect to the second maximum. Therefore, temperature affected the LED efficiency as expected.

\section{Multispectral Camera Results}

From the pictures taken by the camera, it can be seen how the wavelength changed when the temperature of the LED was modified. This effect is observed in the spectral signature of the LEDs (Fig. 2). As an example, Fig. 2c depicts the energy captured by each band of the multispectral camera for the blue LED. Focusing on bands 1, 2 and 3 (centered in 427, 461 and $501 \mathrm{~nm}$, respectively), the wavelength shift can be slightly noticed. In the first place, as temperature increased, part of the emission captured in band 1 was displaced to band 2, which caused growth on its level. While the blue LED was at $10 \mathrm{~mA}$ and $50 \mathrm{~mA}$, the level of bands 1 and 2 barely varied, keeping their levels near 75 and 300, respectively. However, when this LED was at $100 \mathrm{~mA}$ and $130 \mathrm{~mA}$, the level from band 1 to

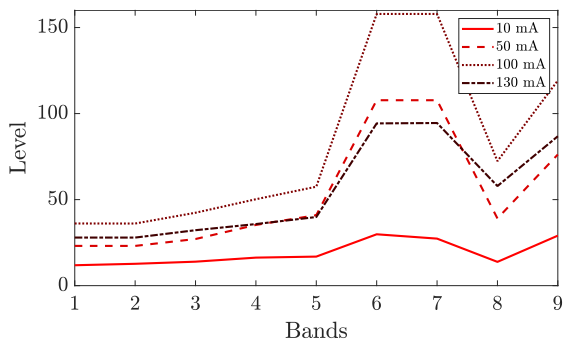

(a) Red LED

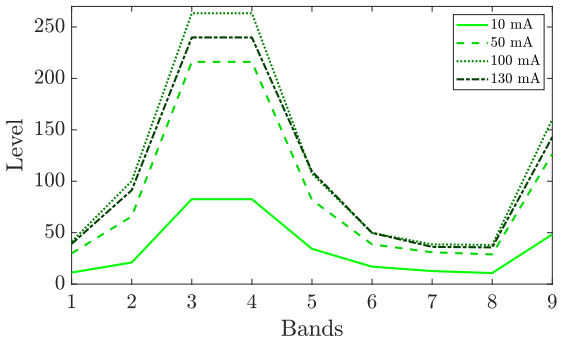

(b) Green LED.

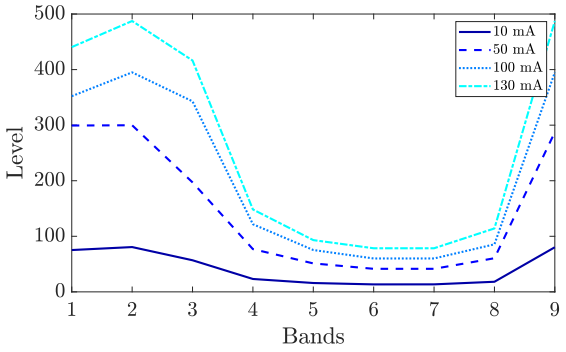

(c) Blue LED

Fig. 2. Spectral signatures of the RGB LED at different temperatures. Solid line, dashed line, dotted line and dash-dot line correspond to $10 \mathrm{~mA}, 50 \mathrm{~mA}$, $100 \mathrm{~mA}$ and $130 \mathrm{~mA}$, respectively.

TABLE II

Condition Number of the Matrices of Spectral Signatures

\begin{tabular}{|c|c|c|c|c|c|c|}
\hline \multirow{2}{*}{ \# channels } & \multicolumn{2}{|c|}{ Best combination (current supplied in mA) } & \multicolumn{3}{c|}{ Condition number (dB) } \\
\cline { 2 - 7 } & $\mathrm{R}$ & $\mathrm{G}$ & $\mathrm{B}$ & $\mathrm{R}$ & $\mathrm{G}$ & $\mathrm{B}$ \\
\hline 2 & 50,130 & 50,130 & 50,100 & 21.9 & 29.9 & 24.5 \\
\hline 3 & $10,50,130$ & $10,100,130$ & $50,100,130$ & 28.9 & 36.2 & 45.4 \\
\hline 4 & $10,50,100,130$ & $10,50,100,130$ & $10,50,100,130$ & 47.9 & 52.3 & 50.7 \\
\hline
\end{tabular}

band 2 increased roughly 100 times (see the slope from band 1 to band 2 in Fig. 2c). The same occurred with bands 2 and 3. This happened noticeably not only due to the red-shifting of the peak wavelength but also to the increment of the FWHM. Apart from the wavelength variation, an evident increment on these bands' level was observed since the temperature was modified by varying the driving current. Besides, the band 9 (panchromatic) increased with current as well, since this band capture data from the entire visible spectrum, being more sensitive in the blue wavelengths. Thus, it can be understood as a power averaging due to the almost flat response of its filter. Similar effects are noted in the spectral signatures of the other tested devices (Figs. 2a and 2b).

The condition number was applied to the matrix of spectral signatures (channel matrix) to mathematically prove that a combined emission of identical devices subject to different temperatures can form separable channels. With the information obtained during the characterization mentioned above, all the available combinations of channel matrices were analyzed. 


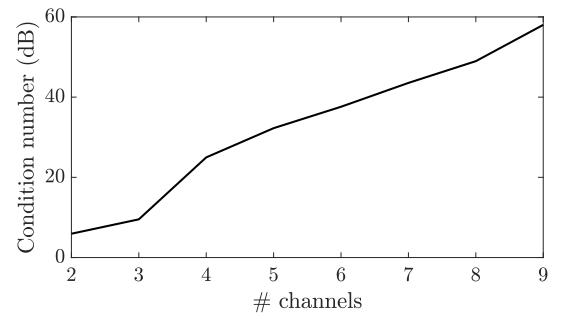

Fig. 3. Minimum condition number obtained for each combination of RGB colors depending on the channels added.

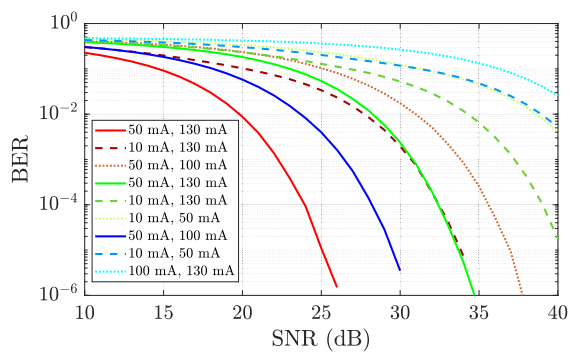

Fig. 4. BER performance of each RGB LED combining two spectral responses at different temperatures. The line color represent the LED color (red, green and blue) and the current values presented in the box are the LEDs driving current for the channel matrix generation. Solid line, dotted line and dashed line correspond to best, worst and average condition number cases, respectively.

Table II highlights the best results depending on the number of target-independent channels. It was observed that the more signatures are added, the more the condition number increases. A striking result that emerged from these data is that the lowest condition numbers were obtained for the red LED. On the other hand, the green LED presented the worst condition number combining four channels. Finally, the blue LED's condition number had a steep growth from two to three channels. This result was obtained for the matrix that included the LED responses at $50 \mathrm{~mA}$ and $100 \mathrm{~mA}$, even though the largest wavelength variation was obtained between $10 \mathrm{~mA}$ (and $50 \mathrm{~mA}$ ) and $130 \mathrm{~mA}$. These values were obtained only by combining spectral signatures of a single color at different temperatures.

Moreover, the best combination of channels in terms of condition number (using the three RGB devices) can be observed in Fig. 3, ranging from 2 to 9 channels (theoretical maximum). As was expected, lower condition numbers were obtained when a combination of different colors was possible, i.e., for 2 and 3 channels. Not surprisingly, as the number of channels increases, so does the condition number. These results offer compelling evidence about the feasibility of providing additional communication channels from a single type of device.

Lastly, the BER performance results are compared in Fig. 4. They correspond to several examples of each RGB color while combining two channels at different currents. These examples show the best, the worst and the average cases regarding the condition number of the matrix $S$. As can be seen, the best results were expectedly obtained for those well-conditioned matrices $(21.9 \mathrm{~dB}, 29.9 \mathrm{~dB}$ and $24.5 \mathrm{~dB}$ for red, green and blue, respectively). On the contrary, in the ill-conditioned matrices $(47.9 \mathrm{~dB}, 52.3 \mathrm{~dB}$ and $50.7 \mathrm{~dB}$ for red, green and blue, respectively), the added noise significantly affected their BER performance, even at high SNR levels.

\section{CONCLUSION}

In this work, the effect of the junction temperature on an LED's emitted spectrum has been experimentally characterized. Furthermore, the spectral variation has been recorded using a multispectral camera. The measurements showed a significant difference between the spectral signature of each LED at different temperatures.

The main objective of this letter was to demonstrate that the aforementioned differences could be used to establish independent communication channels in an OMCC application. In order to assess this, the condition number of the resulting channel matrix was evaluated. It was observed that it is feasible to multiplex several data channels using the same kind of device driven at different average currents (and hence, different temperatures). Additionally, a simulation was developed assuming an OOK transmission to evaluate the OCC system performance. From the BER curves, it could be noted that the better conditioned were the channel matrices, the better the BER performance.

Traditionally, the number of available independent channels using RGB emitters has been assumed to be three (generally, one per device). Nonetheless, this work has demonstrated that using the combination of wavelength-shifted emitters, grasping temperature effects, this limitation that usually damages the transmitted signal can be exceeded, allowing reach up to the number of the receiver's available bands.

\section{REFERENCES}

[1] Short-Range Optical Wireless Communications (802.15.7-2018), Standard 802.15.7-2011, Apr. 2019.

[2] C. Jurado-Verdu, V. Matus, J. Rabadan, V. Guerra, and R. Perez-Jimenez, "Correlation-based receiver for optical camera communications," Opt. Exp., vol. 27, no. 14, pp. 19150-19155, Jul. 2019. [Online]. Available: https://www.opticsexpress.org/abstract.cfm?URI=oe-27-14-19150

[3] H. Liu, S.-H. Lee, and J. S. Chahl, "A multispectral 3-D vision system for invertebrate detection on crops," IEEE Sensors J., vol. 17, no. 22, pp. 7502-7515, Nov. 2017.

[4] M. Rosenberger, "Multispectral edge detection algorithms for industrial inspection tasks," in Proc. IEEE Int. Conf. Imag. Syst. Techn. (IST), Oct. 2014, pp. 232-236.

[5] F. Meriaudeau, V. Paquit, N. Walter, J. Price, and K. Tobin, "3D and multispectral imaging for subcutaneous veins detection," in Proc. 16th IEEE Int. Conf. Image Process. (ICIP), Nov. 2009, pp. 2857-2860.

[6] Y. P. Varshni, "Temperature dependence of the energy gap in semiconductors," Physica, vol. 34, no. 1, pp. 149-154, Jan. 1967.

[7] C. Zhao et al., "The temperature dependence of optical properties of InGaN alloys," Sci. China Phys., Mech. Astron., vol. 55, no. 3, pp. 396-399, Mar. 2012.

[8] J. Park and C. C. Lee, "An electrical model with junction temperature for light-emitting diodes and the impact on conversion efficiency," IEEE Electron Device Lett., vol. 26, no. 5, pp. 308-310, May 2005.

[9] T.-A. Tran and D. C. O'Brien, "Performance metrics for multi-input multi-output (MIMO) visible light communications," in Proc. Int. Workshop Opt. Wireless Commun. (IWOW), Oct. 2012, pp. 1-3.

[10] G. J. Foschini and M. J. Gans, "On limits of wireless communications in a fading environment when using multiple antennas," Wireless Pers. Commun., vol. 6, no. 3, pp. 311-335, Mar. 1998. 